\title{
Electron precipitation events in the lower ionosphere and the geospace conditions
}

\author{
José Henrique Fernandez ${ }^{1, *}$ Emília Correia ${ }^{2,3}$ \\ ${ }^{1}$ Universidade Federal do Rio Grande do Norte (UFRN), Campus Lagoa Nova, Natal, Brazil \\ ${ }^{2}$ Instituto Nacional de Pesquisas Espaciais (INPE), São José dos Campos, Brazil \\ ${ }^{3}$ Centro de Rádio Astronomia e Astrofisica Mackenzie (CRAAM), Universidade Presbiteriana Mackenzie, São Paulo, Brazil
}

Article history

Received October 23, 2012; accepted March 27, 2013.

Subject classification:

Very low frequency amplitude disturbance event, Lightning-induced electron precipitation, Radiation belt electron population, Geospace conditions, Geomagnetic variations.

\begin{abstract}
We present an analysis of localized ionospheric perturbations detected at Comandante Ferraz Brazilian Antarctic Station (Mcllwain parameter $L \sim 2.25$ ) as fast-amplitude variations of very low frequency (VLF) signals transmitted from Hawaii (NPM, at $21.4 \mathrm{kHz}$ ), also known as Trimpi events. The study covers the first six months of 2007, during the period of minimum activity in the $23^{\text {rd }}$ solar cycle. The occurrence of Trimpi events in the Antarctica peninsula region was studied in association with solarwind parameters in the neighborhood of the Earth (geospace), along with the geomagnetic activity level (Ap, Dst indices). The analysis shows that the Trimpi events occurred predominantly during geomagnetically disturbed periods, but they have a more intricate association with the geospace regimes. The events achieve higher occurrence during the recovery phase of some geomagnetic storms, and also show a close association with electron flux enhancements in the belt region, especially those with higher energy. The higher event incidence occurred a few hours after what we call the 'angle bracket' phenomenon: when the solar wind velocity rises simultaneous with a decrease in its density.
\end{abstract}

\section{Introduction}

The reception of very low frequency (VLF) radio waves $(3-30 \mathrm{kHz})$ that propagate in the sub-ionospheric region has been used for many decades as a powerful tool for probing the physical characteristics of the lower ionosphere [e.g., Sechrist 1974, Bainbridge and Inan 2003]. Even if in a localized way, perturbations in plasma density that occur in the reflection altitudes of VLF waves (the D-layer during the day, and E-layer at night) can be detected like phase and/ or amplitude signal sudden variations [Tolstoy and Rosenberg 1982]. A particular phenomenon detected in the VLF signal that is known as localized ionospheric disturbance is produced by the Van Allen belt electron precipitation induced by lightning-generated whistler waves. The characteristic signature of this disturbance on the signal is known as the Trimpi event, and this can only be observed in the ionosphere under night conditions [Helliwell et al. 1973].

In particular, fast variations of the amplitude and phase of VLF signals (called VLF events here) are caused by the Van Allen belt electron precipitation that is induced by lightning-generated (ducted and nonducted) whistler waves, the well-known (classic and oblique) Trimpi events that can be observed only under nighttime conditions, when the ionosphere is not saturated by the solar radiation [Helliwell et al. 1973, Johnson et al. 1999]. These events are produced by wave-particle interactions between the radiation belt electrons and the whistler waves generated by the lightning [e.g., Helliwell et al. 1973, Inan et al. 1978, 1982, Lauben et al. 2001].

On the other hand, statistical analysis of VLF events has shown that they occur predominantly during the equinoxes, similar to semi-annual geomagnetic activity variations [Fernandez et al. 2003]. Some case studies have shown that they occur in closer association with geomagnetically disturbed periods (especially in the recovery phase of some storms) [Fernandez et al. 2006].

To better understand the association between VLF events and the geomagnetic activity, we correlated the occurrence of the events detected at Comandante Ferraz Brazilian Antarctic Station (EACF), located on the Antarctic Peninsula $\left(62^{\circ} 34^{\prime} \mathrm{S}, 58^{\circ} 23.5^{\prime} \mathrm{W}\right.$, McIlwain parameter $\mathrm{L} \sim 2.25$ ) with the conditions of the geospace, during the first six months of 2007.

A better understanding of the ionospheric conditions around the polar regions is one of the main goals of the GNSS Research and Application for Polar Environment (GRAPE) group (2012-2015). The present study attempts to clarify the coupling mechanisms between 


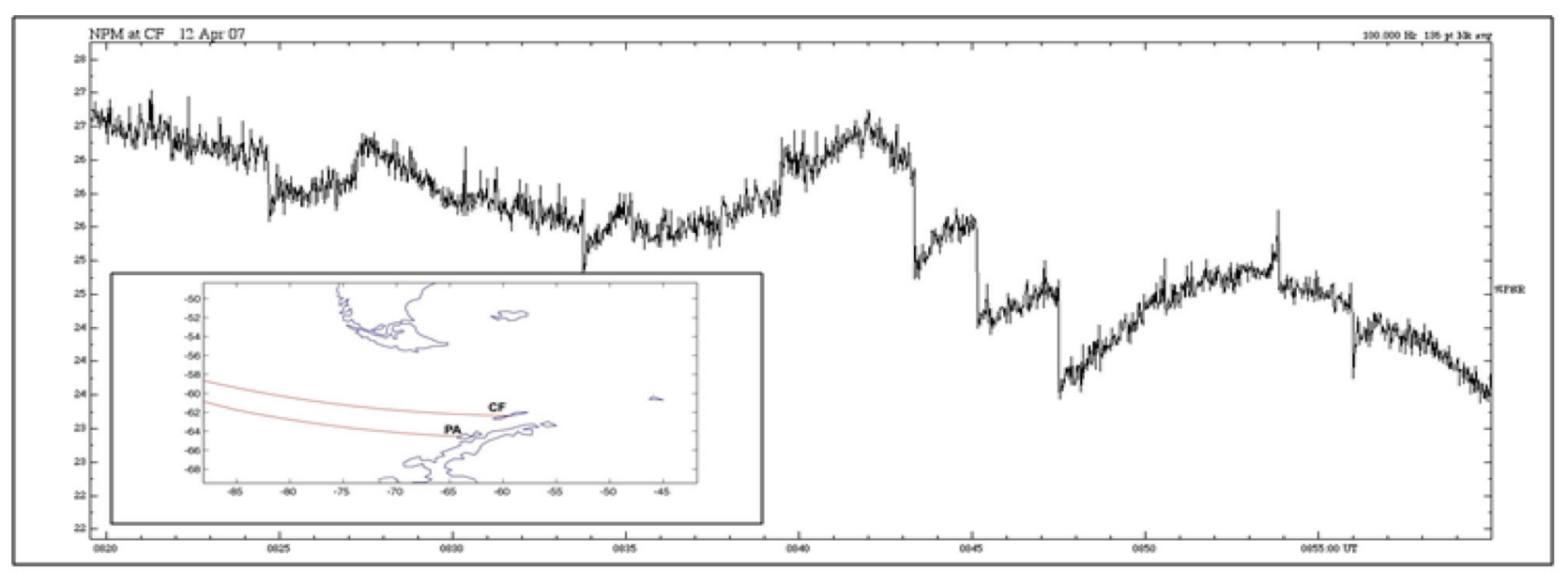

Figure 1. Typical amplitude of the VLF disturbance event profile detected at $21.4 \mathrm{kHz}$ (NPM station, Hawaii) at the Comandante Ferraz Brazilian Antarctic Station (CF). Part of the Great Circle Paths NPM-CF and NPM-Palmer station in the Antarctic Peninsula (PA) is also shown.

the geospace regimes and the lower Earth ionosphere phenomena in the Antarctic peninsula area. The first results of our study indicate the possibility to use the Trimpi activity as a tool to empirically improve the geospace forecasting, while also contributing to feed-back for the ionospheric models, providing lower ionospheric disturbance monitoring in that polar region.

\section{Statistical results of the VLF data analysis and the geomagnetic activity}

Here we analyze the amplitude of the VLF signal transmitted from the NPM Hawaii station (at $21.4 \mathrm{kHz}$; located at $21^{\circ} 25^{\prime} \mathrm{N}, 158^{\circ} 09^{\prime} \mathrm{W}$ ), which is operated by the United States Navy, and detected at EACF. The data were acquired with 10 -ms time resolution using a tunable VLF receiver [Johnson et al. 1999]. About $40 \%$ of the daily VLF data for the period (71 days of the 181 of the entire six months) were inspected in the time interval 00:00 to 12:00 UT. This time window is basically under night-time conditions on the NPM-EACF great circle (GC) path for the majority of the year. The fast amplitude variations $(\sim 3 \mathrm{~s})$ that are followed by a relatively slower recovery time (10-600 s) show amplitude changes above $\sim 1 \%$ full scale range that show the typical 'like-an-exponential' profiles (Figure 1); these were selected as VLF events. To make the data selection, we performed a screening of the days to be analyzed, taking the magnetically quiet days (Dst $>-30 \mathrm{nT}$ ) and the magnetically active days (Dst $\leq-30 \mathrm{nT}$ ) as reference, identified by the Dst index reported for the period.

The selected VLF data refer to the amplitude variation, time of the occurrence (defined as the beginning of the event), onset time, inter-event time (to define an event occurrence rate), and duration time of each identified VLF event. The amplitude variation was taken as a percentage of the full scale range, with the threshold of the signal level detection at $0 \%$, and the saturation level at the EACF site at $100 \%$. The onset time is defined as the time interval between the start of the abrupt drop in the amplitude signal and the beginning of the recovery phase, which is the interval that is also used to measure the amplitude variation.

Figure 2 shows the monthly number of VLF events (day-by-day) plotted in association with the Ap and Dst geomagnetic indices obtained from the World Data Center for Geomagnetism, Kyoto, Japan. Table 1 shows the monthly averages of the main event characteristics, as mentioned above. In Figure 2, it can be seen that the VLF events occurred preferentially during the geomagnetically disturbed days, and the comparison of the monthly data shows good correlation between the occurrence of VLF events and four distinct geomagnetic

\begin{tabular}{|c|c|c|c|c|c|c|}
\hline Monthly average & Jan & Feb & Mar & Apr & May & June \\
\hline Time inter-events (s) & 169 & 128 & 3204 & 1708 & 2064 & 600 \\
\hline Onset (s) & 05 & 06 & 03 & 03 & 04 & 02 \\
\hline Amplitude variation (\%FSR) & 1.45 & 0.91 & 0.92 & 1.16 & 1.05 & 1.00 \\
\hline Quite days (number of events) & 0 & 17 & 31 & 0 & 10 & 5 \\
\hline Total detected events & 109 & 653 & 181 & 325 & 120 & 5 \\
\hline
\end{tabular}

Table 1. Statistical properties of the VLF disturbance event analysis, January to June 2007. \%FSR, percentage full scale range. 

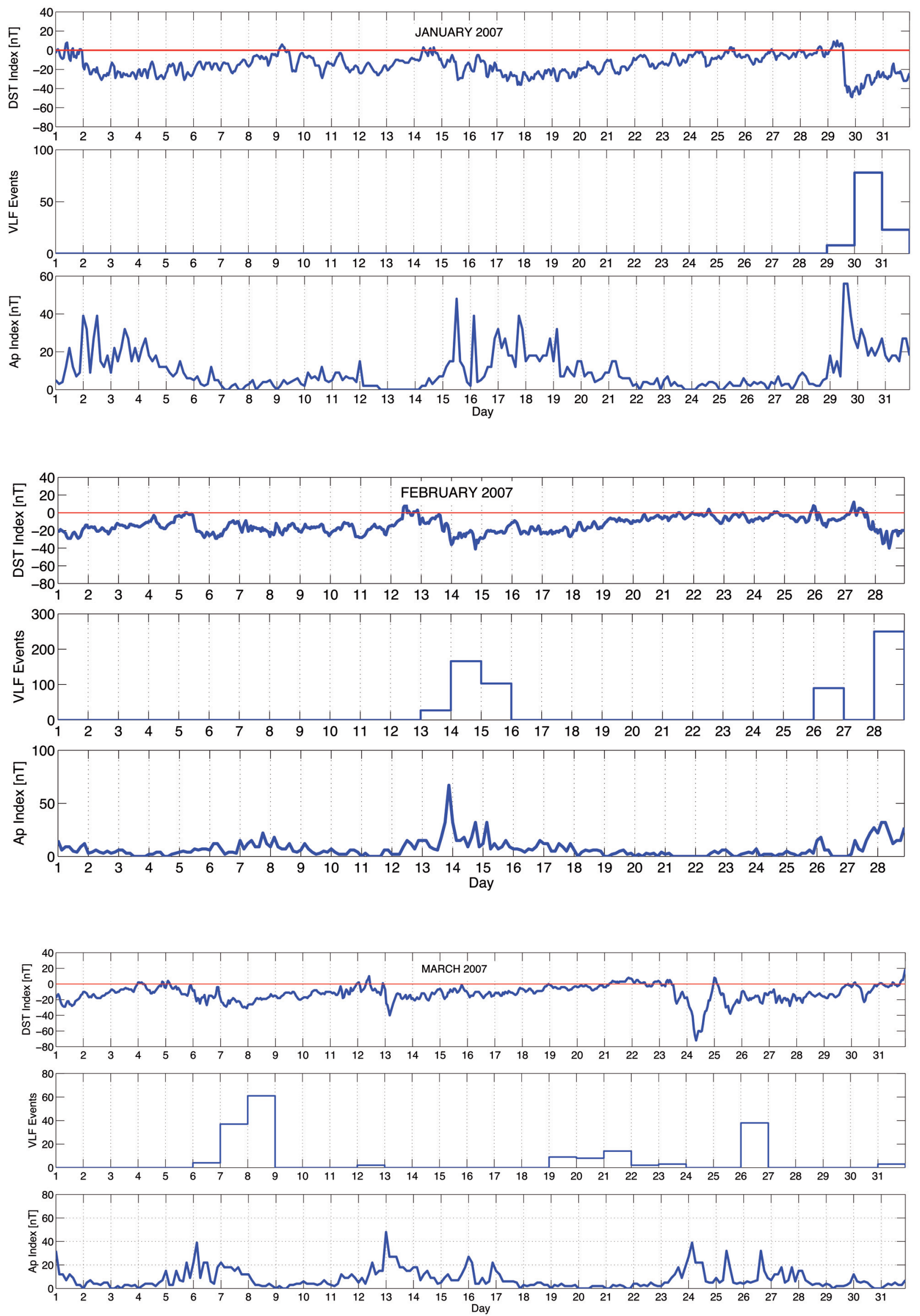

Figure 2 (continues on following page). Time profile of the disturbance events on the VLF amplitude signal, and the Dst and Ap magnetic indices for January to June 2007, as indicated. 

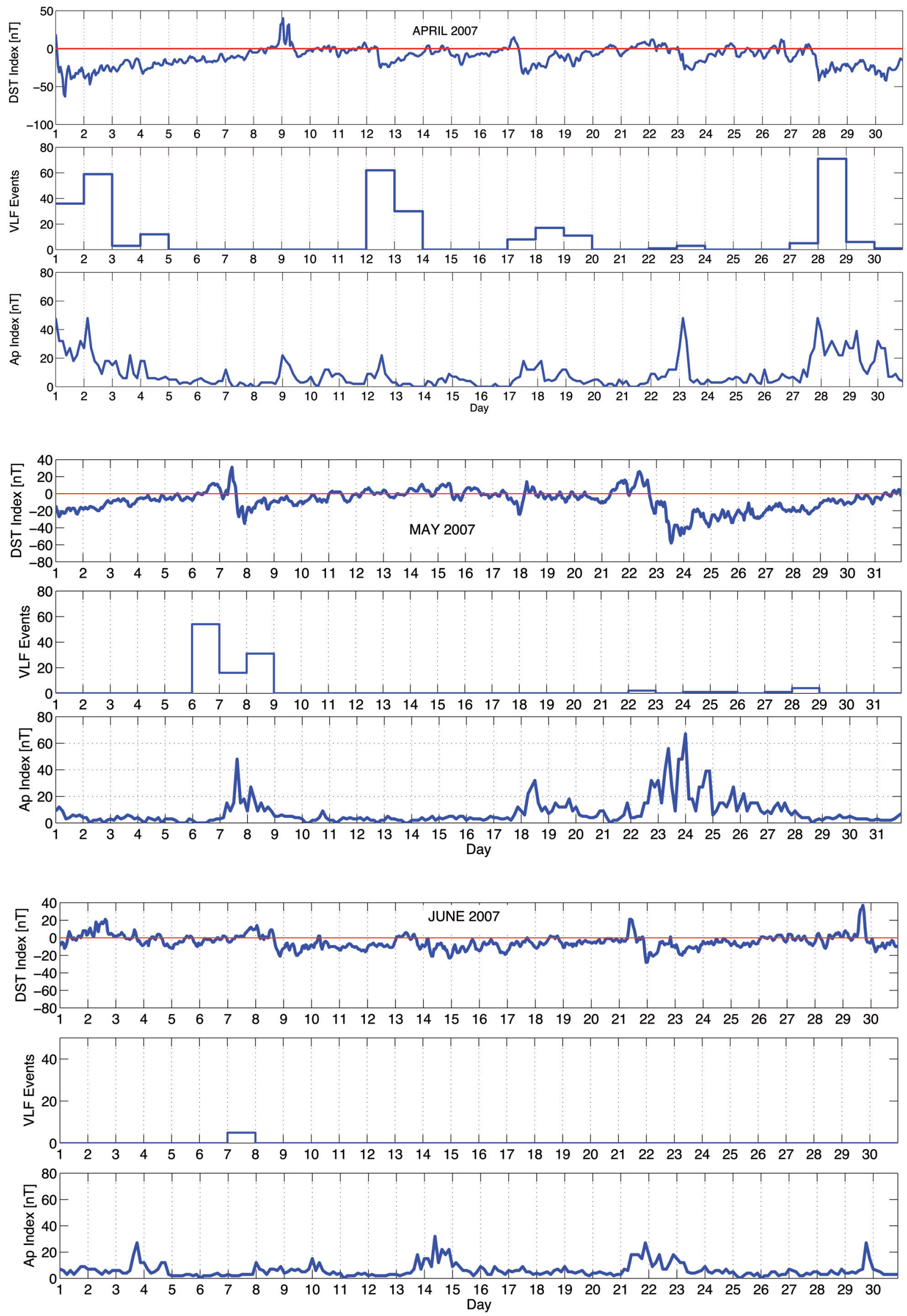

Figure 2 (continues from previous page). Time profile of the disturbance events on the VLF amplitude signal, and the Dst and Ap magnetic indices for January to June 2007 , as indicated. 

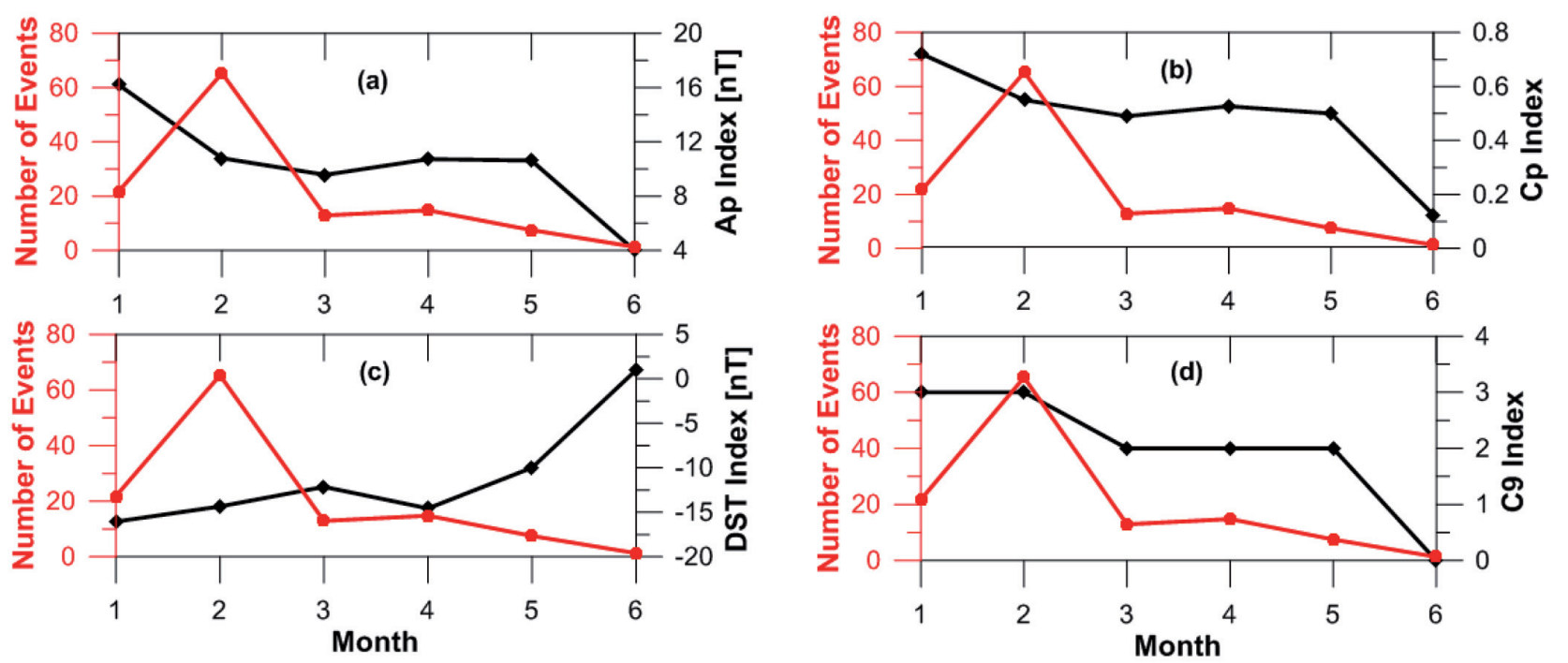

Figure 3. Comparison between the monthly VLF event occurrence rate and: (a) the Ap index; (b) the Cp index; (c) the Dst index; and (d) the C9 index, from January to June (months 1-6) 2007.

indices for each month (Figure 3), except in January. Just a few of the days ( 8 days of the total of 71 days; i.e., $11.3 \%$ of the total analyzed data) presented Trimpi incidences during the relatively quiet geomagnetic regimes $(>-15 \mathrm{nT})$. There is also a small difference between the monthly incidence curve of the VLF events and each curve of the magnetic indices plotted in Figure 2. To explore the reasons for these differences, we compared the monthly averages of the Trimpi events, the day-by-day averages, and the Ap, Dst, Cp and C9 geomagnetic indices (Figure 3). For the curves in the Figure 3 , we took just the averages over the selected days in the analysis, screened as described previously.

The anomalous high Trimpi occurrence that is observed in February, in comparison with previous analyses [e.g., Fernandez et al. 2003], can be explained by the high lightning rate for the same period (which was unfortunately not investigated in detail here). The low occurrence in January can be explained because the ionospheric conditions, which were under day-time due to the summer season (saturation), for almost all of this month. Only at the end of January, coincident with the observed high Trimpi activity, does the sub-antarctic ionosphere start to have a few hours of night-time conditions. Figure 3 also shows that there is a better correlation between the VLF event rate and the $\mathrm{Cp} / \mathrm{C}^{1}$ index (again, in the interval from February to June). This might be explained because $\mathrm{Cp}$ is a qualitative estimate of the overall level of magnetic activity for the day. Cp is determined by the daily sum of eight Ap amplitudes, and hence it is better for average applications. Moreover, the $\mathrm{Cp}$ index also has a pronounced 27-day

1. The $\mathrm{C} 9$ index converts the 0 to 2.5 range of the $C p$ index to one digit between 0 and 9 , as defined by the appropriated Table [e.g., Mayaud 1980, Siebert and Meyer 1996]. tendency recurrence, and it is strongly associated with coronal mass ejection arrivals at the geospace.

The strong association of the VLF event occurrence with the geomagnetic activity, especially during the storm recovery phase, can be attributed to the increase in the radiation belt population due the arrival/ capture of energetic electrons and protons.

Table 1 shows that February is the month with the greatest VLF event occurrence, and also with the longest onset time and the minimum time interval between neighboring events. When the occurrence rate of VLF events is high, there is a superposition of single events, and it is impossible to separate these. This causes the apparently longer onset time. The elevated rates of Trimpi occurrence might be due to more efficient precipitation processes, which means that there are more whistler waves, an enhanced gyro-resonant electron population, and longer resonant time available in the radiation belt region. A way to evaluate the duration of the wave-particle resonant time is the estimation of the precipitating electron fluxes through the measurement of the VLF event duration time. This analysis shows that the VLF events have a typical profile, although with a range of varied shapes. The event profile is similar to non-symmetric stalactite (negative ones) or stalagmite (positive ones) in the received amplitude signal. It is non-symmetric because of the fast onset and the relatively longer (like-an-exponential) recovery times. While the total duration of the VLF event (the measure of the basis of the stalactite/ stalagmite) is related to the flux of the precipitation particles, the amplitude variation of a single event (the length of the stalactite/ stalagmite) is related to the energy of the particles (penetration depth in the atmosphere) and the distance from the VLF GC path. So it is possible to observe a wide range of event profiles, some with large amplitude variations, although 

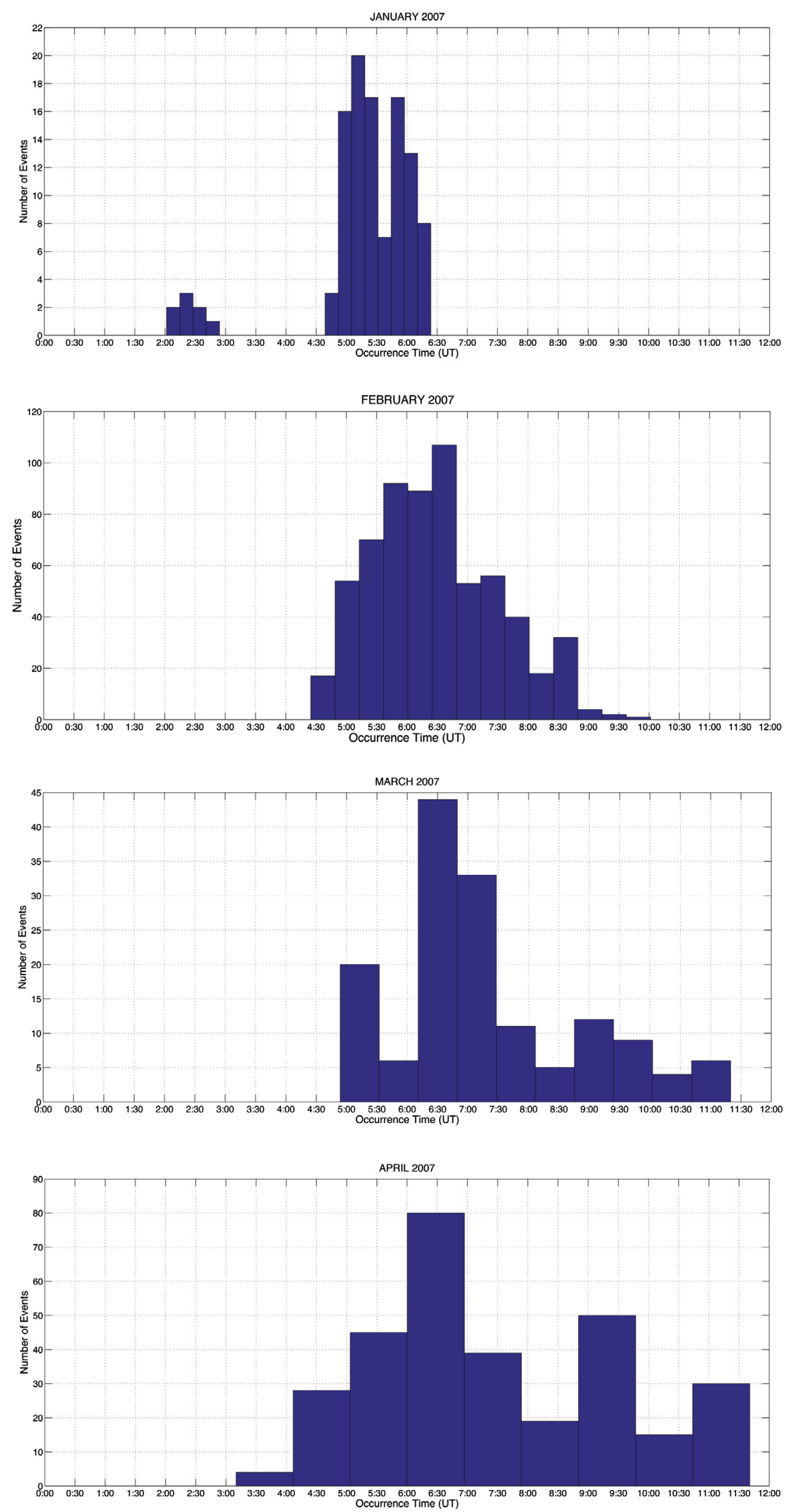

Figure 4 (continues on following page). VLF event occurrence time histograms for January to May 2007, as indicated. June is not included because of the low event occurrence. 


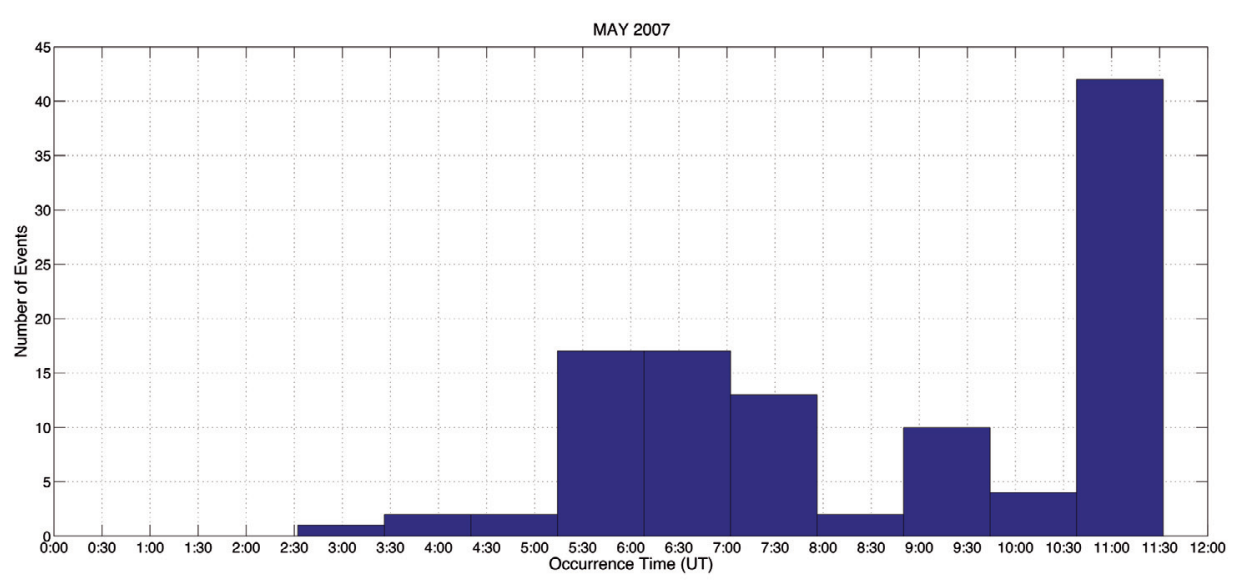

Figure 4 (continues from previous page). VLF event occurrence time histograms for January to May 2007, as indicated. June is not included because of the low event occurrence.

\begin{tabular}{lcccc}
\hline Year 2007 & Period & 'Bracket' phenomenon & VLF event occurrence ${ }^{\star}$ & Geomagnetic activity $^{\star \star}$ \\
\hline Case A & Jan 29-31 & Yes & High & Dst peak $(-50 \mathrm{nT})$ \\
Case B & Feb 26-28 & Yes & Very high & Dst peak $(-40 \mathrm{nT})$ \\
Case C & Mar 19-26 & Yes & Moderate & Dst peak $(-70 \mathrm{nT})$ \\
Case D & Apr 12-13 & No & Moderate & Dst peak $(-30 \mathrm{nT})$ \\
Case E & May 4-8 & Yes & Moderate & Dst peak $(-40 \mathrm{nT})$ \\
Case F & May 22-28 & Yes & Low & Dst peak $(-60 \mathrm{nT})$
\end{tabular}

Table 2. Summary of the cases of interest. ${ }^{\star}$, Very high occurrence is $>200$ events per day, high occurrence is $\sim 80-100$ events per day, moderate occurrence is $40-60$ events per day, and low occurrence is $<10$ events per day; ${ }^{\star \star}$, values rounded.

short time duration, and some with small amplitude variations, but long duration.

The occurrence time of the Trimpi events was also investigated. Figure 4 shows the month-by-month histograms of the period considered. The results obtained here confirm those obtained by Fernandez et al. [2003], who analyzed data from the period of April 1996 to March 1997 (also during a solar minimum period), with a mean incidence peak at 7:00 UT, and a secondary peak tending to be formed at $\sim 10: 30 \mathrm{UT}$ in the course of the year.

\section{Geospace conditions during the occurrence peri- ods of the VLF events}

To better understand the close association between Trimpi events and geomagnetic activity, we analyzed the geospace conditions during some cases of interest in the first six months of the year 2007.

We selected six different sets of days, named as Case A to Case F (see Table 2). Case A corresponds to the Trimpi incidence period from January 29 to 31. Figure 5 shows the corresponding geospace conditions obtained for Case A from the instruments aboard the ACE satellite (http:/ / www.srl.caltech.edu / ACE / ASC / level2 / index.html). Figure 5 shows that the enhancement of the VLF event occurrence is accompanied by an expressive $^{2}$ disturbance in the interplanetary magnetic field (IMF). This disturbance is evident in the module of the total magnitude $(|\mathrm{B}|)$ and as fluctuations of the $z$ component $\left(\mathrm{B}_{\mathrm{z}}\right)$, the latter being a proxy for the reconnections between the Earth field and the IMF that drives particle capture to the geospace.

A few hours before the IMF disturbance peaks, it is possible to observe an enhancement in the electron fluxes, from the top of Figure 5, especially in the higher energy channel (175-312 keV). Jointly with the peak of the IMF disturbances, and approximately also with the peak in the observed electrons fluxes, a remarkable phenomenon occurs: the solar wind proton velocity and density rapidly change simultaneously, with the velocity rising and the density decreasing. Referring to the bottom plot of Figure 5, when plotted together, these curves appear to form a sort of 'angle bracket', followed by a plateau region. Just after this angle is finished, i.e., at the beginning of the plateau, the Trimpi event occurrence rises.

\footnotetext{
2. For 'expressive' we mean an increase greater than two times the standard deviation of the immediately previous temporal series fluctuation. This becomes evident when observed in the plot; e.g., in Figure 5.
} 


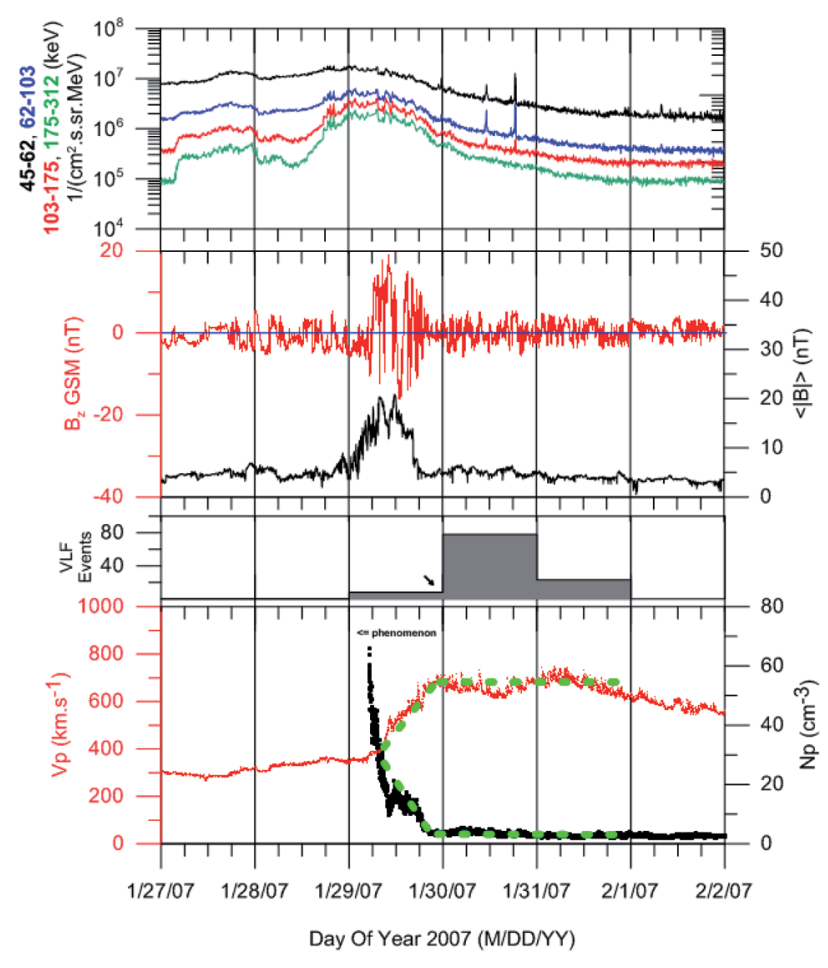

Figure 5. Case A: Jan 29-31. ACE data. Top: Electron fluxes (Ep) from EPAM. Middle: IMF ( $|\mathrm{B}|$ and Bz) from MAG. Bottom: Solar wind proton density $(\mathrm{Np})$ and velocity $(\mathrm{Vp})$ from SWEPAM.

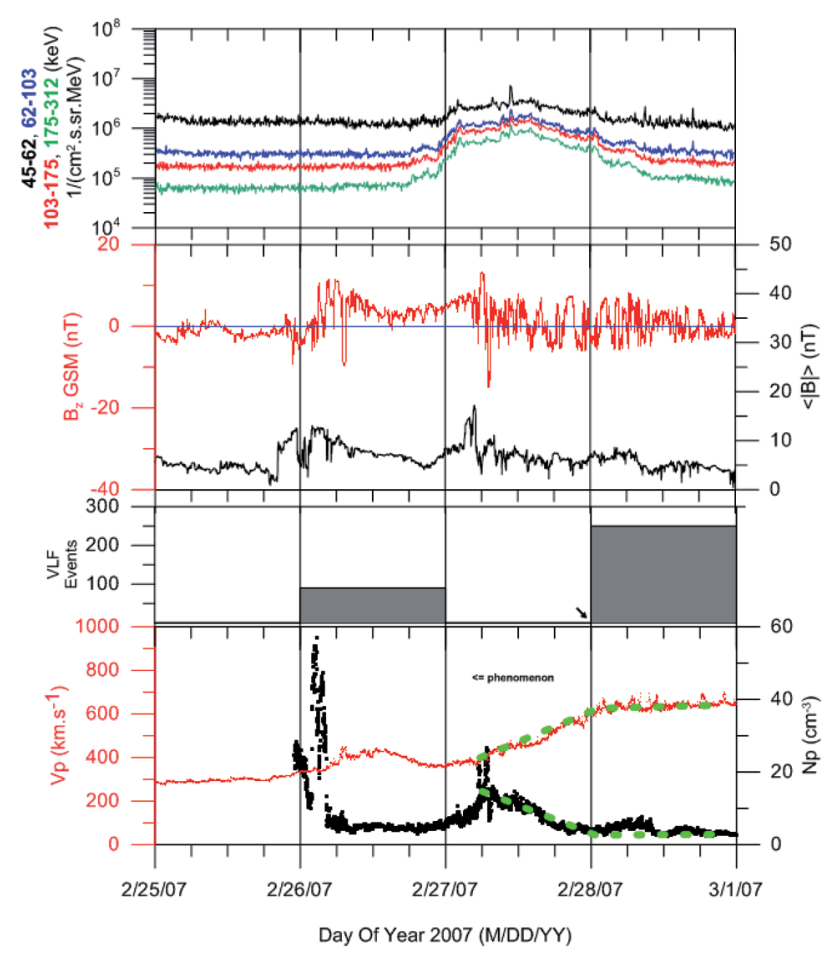

Figure 6. As for Figure 5, for Case B: Feb 26-28.

The same phenomenon is seen in Figures 6, 7 and 9, which correspond to Case B (February 26 to 28), Case C (March 24 to 26) and Case E (May 7 to 8), respectively, except for the absence of the enhanced electrons fluxes in Case C (Figure 7). In Case D (April 12 to 13), which is plotted in Figure 8, despite the magnetic activity reported by the Dst index (although relatively less expressive in the
Ap index) and also the observed Trimpi occurrence (relatively high), the geospace conditions were much more quiet (only a small but abrupt fluctuation in the IMF Bz was seen, from north to south, on April 12). On the other hand, on April 14 (also shown in Figure 8), the range of the wind velocity variation was not expressive enough, and also there was a gap in the density data. It is important to note that this is the opposite situation to that described previously, and also the flat region was not configured. This might explain the absence of VLF events in the subsequent days (April 14 to 16).

Finally the most curiously case is Case F in Figure 10. Here, all of the conditions were favorable to lead to a high Trimpi occurrence rate, namely: (a) high magnetic activity reported, from both the Dst and the Ap indices, which actually reached the highest disturbed levels for the whole of the period studied; (b) all of the characteristics of our 'angle bracket' phenomenon were also present. Large variations in the $|\mathrm{B}|, \mathrm{B}_{\mathrm{z}}$ and in all of the electron fluxes were observed, the solar wind proton velocity was increasing, and its density was decreasing, as expected. However, even with all of these conductive scenario conditions, there was only a poor VLF event rate in this interval.

This shows that the occurrence of the electron precipitation events from geospace to the upper Earth atmosphere is somewhat more complex, and evolves the coupling of different phenomena at different levels of efficiency. Certainly, the geospace conditions (electron fluxes, $B_{z}$ fluctuations, solar wind regimes) influence the noted Trimpi event occurrence, as observed in most cases, but the LEP phenomenon also has the ' $\mathrm{L}$ ' counterpart (the whistler mode waves produced by lightning, which were not investigated here). We conclude that there is a combination of factors that leads to a higher VLF disturbance event rate, and we are sure that all of the geospace conditions contribute significantly, even if they are necessary but not sufficient conditions for Trimpi occurrence. All of the main conclusions about the study of these cases are summarized in Table 2 .

\section{Concluding remarks}

The present study shows strong evidence that the geomagnetic activity and geospace weather regimes can be partners in electron precipitation events detected as localized ionospheric disturbances, the Trimpi events, through the use of VLF subionospheric propagating signals. It is possible to identify a close association between the geomagnetic activity and the VLF event occurrence. The electron precipitation from the Van Allen radiation belts to the upper Earth atmosphere is nowadays described as a phenomenon that evolves the wave-particle interaction; i.e., the resonance between the whistler 


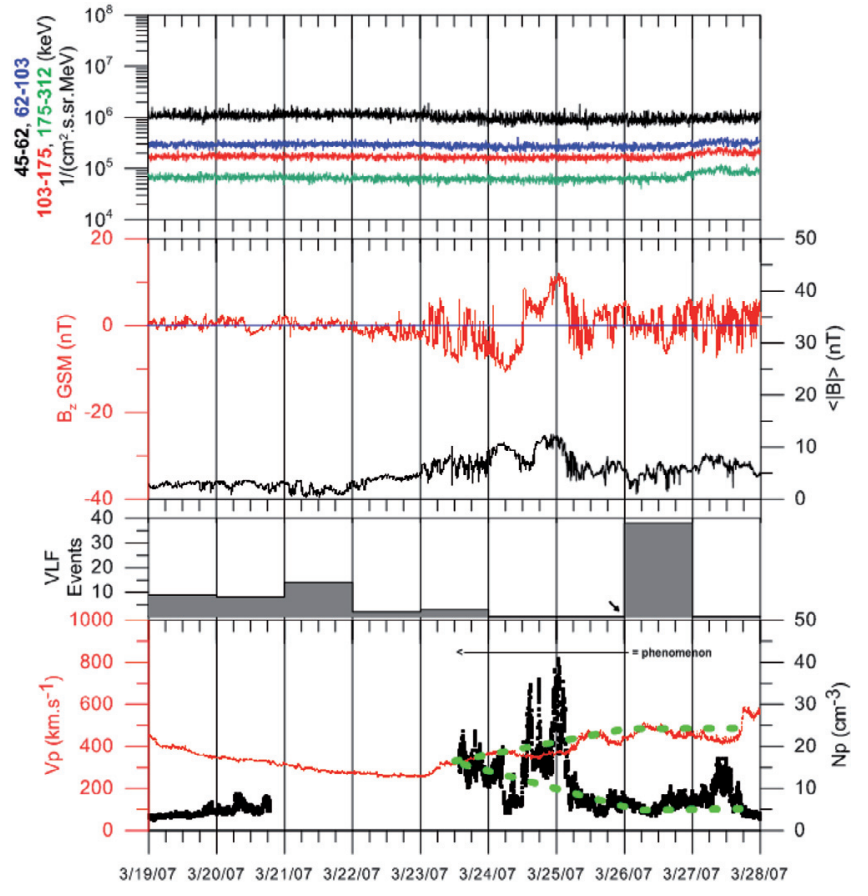

Day Of Year 2007 (M/DD/YY)

Figure 7. As for Figure 5, for Case C: Mar 19-26.

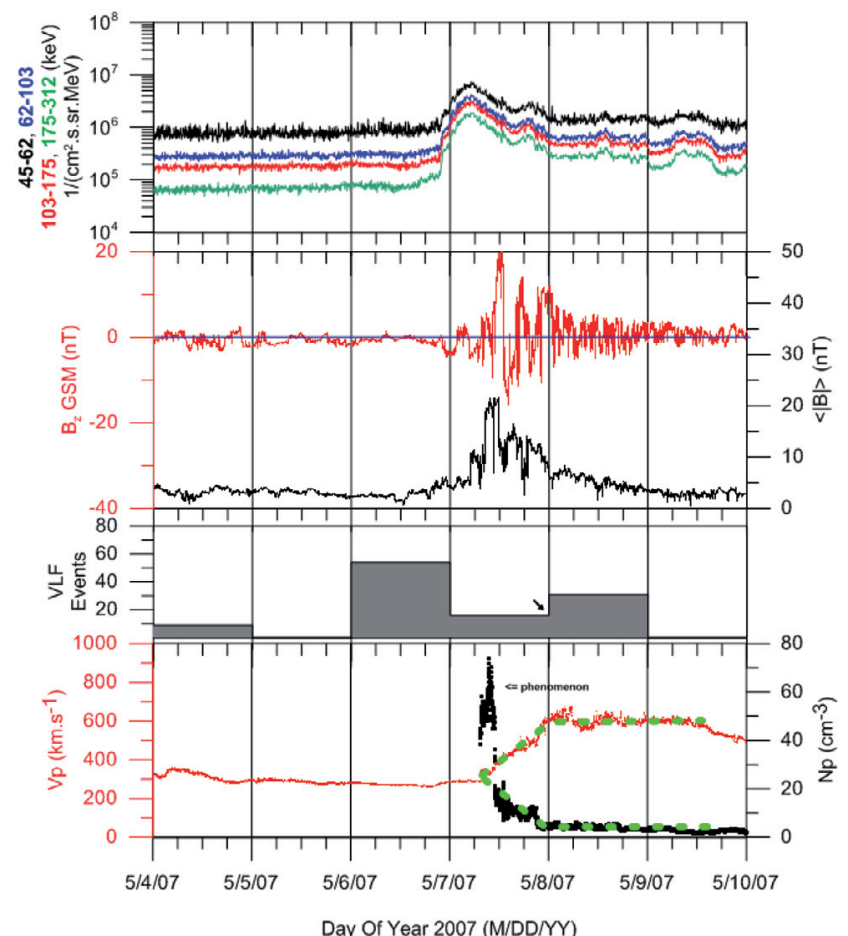

Figure 9. As for Figure 5, for Case E: May 4-8.

mode waves (lightning generated) and the gyro-resonant belt-trapped electrons. This means that any factor that can affect the electron population, the time, and/or the stability of the wave-particle interaction, and finally the 'amount' of interacting waves available in the geospace, will guide the resulting number of VLF events detected. Here, we indeed show that changes in the geo-

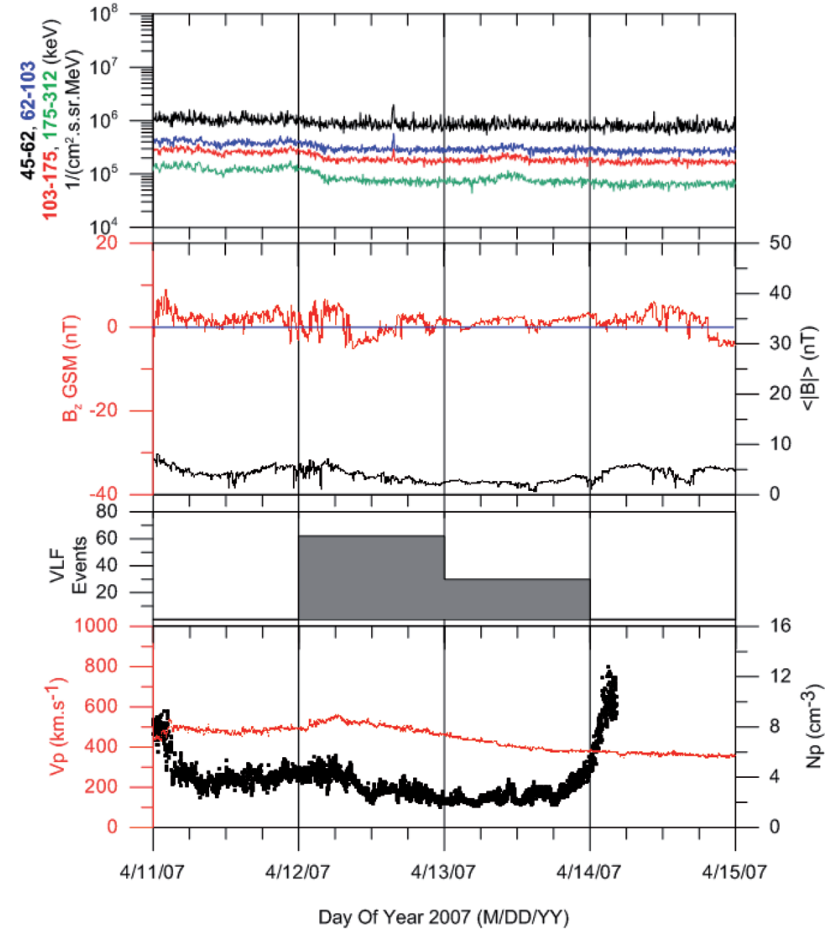

Figure 8. As for Figure 5, for Case D: Apr 12-13.

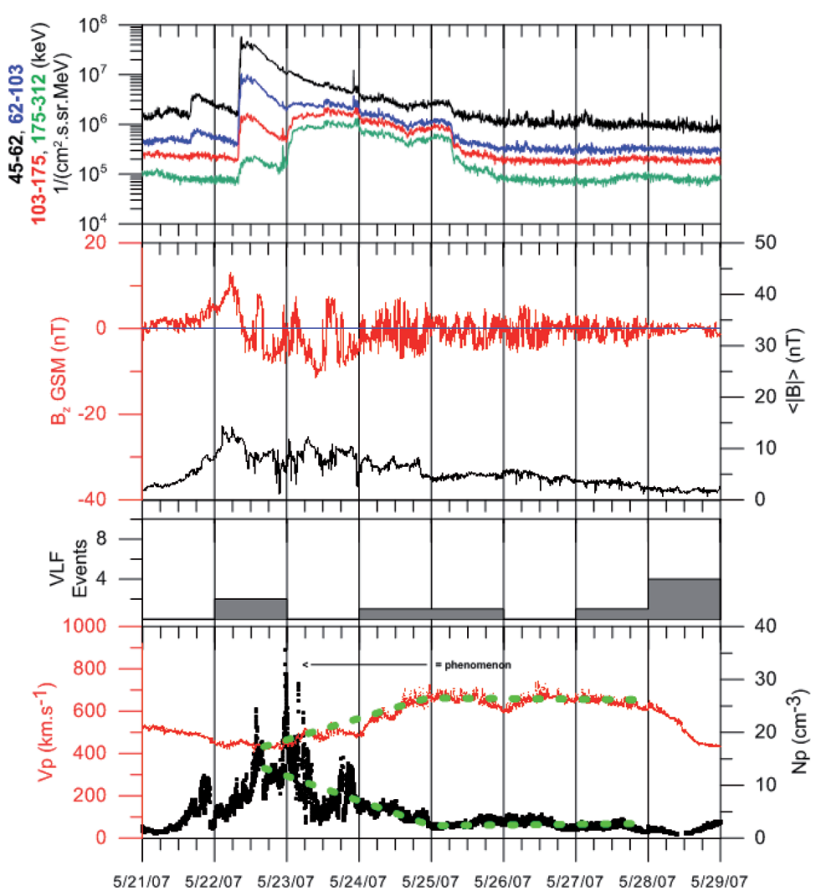

Day Of Year 2007 (M/DD/YY)

Figure 10. As for Figure 5, for Case F: May 22-28.

space regimes associated with geomagnetic variations very often lead to increased rates of VLF event occurrence. The large fluctuations observed at IMF, which point the $\mathrm{B}_{\mathrm{z}}$ component to the north and south with higher time frequencies and high amplitude variations, favor the magnetic reconnection, and as a consequence, it drives to the capture of plasma fluxes in the belt re- 
gions, enhancing the trapped particle population. Another possible explanation is the generation of magnetospheric waves in the above-described processes. Kavanagh and Denton [2007] cited Green et al. [2004] and suggested that enhanced precipitation fluxes might be due to interactions with these waves, although to better explore this aspect, a parallel analysis of the lightning rates is also essential (whistler generation).

Acknowledgements. The authors wish to thank the World Data Center for Geomagnetism at Kyoto University, Japan, for the geomagnetic indices, and the ACE Satellite group for the solar wind data used in this study. This study was partially support by the CNPq/PROANTAR Brazilian Antarctic Program. We also thank the reviewers for their comments/ suggestions that helped us to improve our manuscript.

\section{References}

Bainbridge, G., and U.S. Inan (2003). Ionospheric D region electron density profiles derived from the measured interference pattern of VLF waveguide modes, Radio Sci., 38, 16-21.

Fernandez, J.H., L. Rizzo Piazza and P. Kaufmann (2003). Trimpi occurrence and geomagnetic activity: Analysis of events detected at Comandante Ferraz Brazilian Antarctic Station ( $\mathrm{L}=2.25)$, J. Geophys. Res., 108, 1041-1048.

Fernandez, J.H., J-P. Raulin, E. Correia and C.G.M. Brum (2006). Lightning-induced electron precipitation (LEP) events versus geomagnetic activity: a probe tool to re-evaluate the electron radiation belt loss mechanisms, In: $2^{\text {nd }}$ UN/NASA Workshop on International Heliophysical Year and Basic Space Science, Proceedings of the conference, Indian Institute of Astrophysics, Bangalore, India, 108.

Green, JC, T.G. Onsager, T.P. O Brien and D.N. Baker (2004). Testing loss mechanisms capable of rapidly depleting relativistic electron flux in the Earth's outer radiation belt, J. Geophys. Res., 109, 2156-2202.

Helliwell, R.A., J.P. Katsufrakis and M.L. Trimpi (1973). Whistler-induced amplitude perturbation in VLF propagation, J. Geophys. Res., 78, 4679-4701.

Inan, U.S, T.F. Bell and R.A. Helliwell (1978). Nonlinear pitch angle scattering of energetic electrons by coherent VLF waves in the magnetosphere, J. Geophys. Res., 83, 3235-3253.

Inan, U.S, T.F. Bell and H.C. Chang (1982). Particle precipitation induced by short-duration VLF waves in the magnetosphere, J. Geophys. Res., 87, 6243-6264.

Johnson, M.P., U.S. Inan and D.S. Lauben (1999). Subionospheric VLF signatures of oblique (nonducted) whistler-induced precipitation, Geophys. Res. Lett., 26, 3569-3572.

Kavanagh, A.M., and M. Denton (2007). High-speed solar-wind streams and geospace interactions, A\&G Meeting Report, 48, 6.24-6.26.

Lauben, D.S., U.S. Inan and T.F. Bell (2001). Precipitation of radiation belt electrons induced by obliquely propagating lightning-generated whistlers, J. Geophys. Res., 106, 29745-29770.

Mayaud, P.N. (1980). Derivation, meaning and use of geomagnetic indices, Geophys. Monogr., Am. Geophys. Union, Washington, D.C., 22, 154 pp.

Sechrist, C.F. (1974). Comparison of techniques for measurement of D region electron densities, Radio Sci., 9, 137-149.

Siebert, M., and J. Meyer (1996). Geomagnetic activity indices, In: W. Dieminger, G.K. Hartmann and R. Leitinger (eds.), The Upper Atmosphere: Data Analysis and Interpretation, Springer, Berlin/Heidelberg/New York, 887-911.

Tolstoy, A., and T.J. Rosenberg (1982). The influence of localized precipitation-induced D-region ionization enhancements on subionospheric VLF propagation, Geophys. Res. Lett., 9, 563-566.

\footnotetext{
${ }^{\star}$ Corresponding author: José Henrique Fernandez, Universidade Federal do Rio Grande do Norte (UFRN), Campus Lagoa Nova, Natal, Brazil; email: jhfernandez@ect.ufrn.br or jhenrix@gmail.com.

(C) 2013 by the Istituto Nazionale di Geofisica e Vulcanologia. All rights reserved.
} 